R. L. P. Santos - M. C. Zillikens $\cdot$ F. R. Rivadeneira •

H. A. P. Pols - B. A. Oostra - C. M. van Duijn •

Y. S. Aulchenko

\title{
Heritability of fasting glucose levels in a young genetically isolated population
}

Received: 15 July 2005 / Accepted: 22 November 2005 / Published online: 8 February 2006

(C) Springer-Verlag 2006

\begin{abstract}
Aims/hypothesis: The heritability of fasting glucose levels in Northern European populations has been examined previously in twins and samples of small pedigrees. In this study the heritability of fasting plasma glucose (FPG) was estimated in participants in the Erasmus Rucphen Family study, who were members of a single pedigree from a young genetic isolate. We also studied the relationship between FPG and components of the metabolic syndrome. Methods: FPG, lipid, blood pressure and body composition measurements were completed for 852 participants without diabetic medication. The most significant predictors of FPG were used as covariates in heritability estimation. The sibship effect, which is a composite of genetic dominance and shared early-life environmental effects, was included as a random effect. Results: The age- and sex-adjusted heritability of $\log$ normal-transformed FPG was $36.6 \%$. When further adjusted for metabolic risk factors, namely body composition parameters, systolic blood pressure, triglycerides and cholesterol:HDL ratio, the heritability estimate rose to $42.8 \%$. After adjustment for the sibship effect, the additive
\end{abstract}

R. L. P. Santos · M. C. Zillikens · F. R. Rivadeneira •

H. A. P. Pols - B. A. Oostra - C. M. van Duijn ·

Y. S. Aulchenko $(\bowtie)$

Genetic Epidemiology Unit,

Department of Epidemiology and Biostatistics,

Erasmus MC Rotterdam,

P.O. Box 1738, 3000 DR Rotterdam, The Netherlands

e-mail: i.aoultchenko@erasmusmc.nl

Tel.: +31-10-4087362

Fax: +31-10-4089406

M. C. Zillikens · F. R. Rivadeneira · H. A. P. Pols Department of Internal Medicine, Erasmus MC Rotterdam, Rotterdam, The Netherlands

B. A. Oostra

Department of Clinical Genetics, Erasmus MC Rotterdam,

Rotterdam, The Netherlands

Y. S. Aulchenko

Institute of Cytology and Genetics SD RAS,

Novosibirsk, Russia component of heritability was estimated to be $28.3 \%$ (ageand sex-adjusted) and $24.9 \%$ (full model). Conclusions/ interpretation: Genes control a significant proportion of the variance in FPG levels. Adjustment for other metabolic risk factors did not substantially change the heritability estimate, which suggests that a large part of the variance in FPG levels is due to genes that act through pathways that are independent of those controlling body composition, blood pressure and lipid levels.

Keywords Erasmus Rucphen Family study · ERF . Fasting glucose $\cdot$ Genetic isolate $\cdot$ Heritability Metabolic syndrome

Abbreviations ERF: Erasmus Rucphen Family study . FPG: fasting plasma glucose - DEXA: dual-energy X-ray absorptiometry $\cdot$ FM: fat mass - FMI: fat mass index · LMI: lean mass index $\cdot \mathrm{Tr} / \mathrm{l}$ : trunk/legs fat ratio · SBP: systolic blood pressure - Trig: triglycerides . TC/HDL: total cholesterol/HDL cholesterol ratio

\section{Introduction}

The prevalence of diabetes mellitus is rising rapidly worldwide. In 1995, 135 million adults were affected with diabetes globally, and this number is estimated to reach 300 million by 2025 [1]. An elevated fasting glucose level is the most commonly used method of diagnosing diabetes. Other indices, such as abdominal obesity, hypertension, hyperlipidaemia and hypertriglyceridaemia, are also associated with elevated fasting glucose. These phenotypes tend to cluster together as the metabolic syndrome [2]. The prevailing hypothesis is that the different metabolic syndrome components are, at least in part, caused by common genes [3]. However, these metabolic states are also greatly influenced by environmental factors, such as intrauterine environment, diet and physical activity $[4,5]$.

As an initial step in the search for genes that cause altered metabolic states, heritability studies have been conducted for various traits, particularly for diabetes and its 
endophenotypes. The heritability estimate shows how much of the phenotypic variance is attributable to the average effects of genes [6]. For fasting glucose, heritability studies in Northern Europe have mostly been done using twin registers, in which heritability estimates ranged from 38 to $51 \%$ [7-9]. Heritability estimates tend to be higher among twins than in extended pedigrees because of greater similarities in early-life environment and behavioural patterns among twins. In family studies, heritability estimates vary widely $(8-72 \%)$, which may be partly due to ascertainment bias $[10,11]$. In a study of 89 randomly ascertained pedigrees, in which variance due to childhood household was also measured, the heritability of fasting glucose was $18 \%$ after adjustment for age, sex, BMI, and smoking [12].

Here we report the heritability of fasting plasma glucose (FPG) in a large extended pedigree with 6,592 members, among whom 951 living subjects were characterised for different metabolic parameters. Ascertainment of the sample was based not on disease status, but on the participants' relation to 20 couples who lived in the area in the second half of the nineteenth century. The presence of numerous extended relative pairs allowed the distinction between additive genetic and sibship effects, the latter being a composite of genetic dominance and shared early-life environment effects. Phenotypic description of this population includes a serum lipid profile and fat and lean mass measurements using dual-energy X-ray absorptiometry (DEXA), thus enabling us to study the relation between FPG and a number of metabolic syndrome features, such as obesity, hypertension and hypertriglyceridaemia.

\section{Subjects and methods}

\section{Study sample}

Our study sample was based on a young, genetically isolated population in the southwest of The Netherlands. This community was founded by $\sim 150$ Caucasians in the mid-eighteenth century. Since then there has been minimal migration from the community and few marriages with people from outside the community. Today roughly 20,000 people live within the community and its neighbouring areas. For this population, an extensive genealogical database is available, which was reconstructed through detailed church and municipal records.

The study protocol was approved by the medical ethics board of Erasmus MC Rotterdam. All investigations were carried out in accordance with the Declaration of Helsinki. The Erasmus Rucphen Family (ERF) study was designed to sample approximately 3,000 people who are descendants of 20 couples from the second half of the nineteenth century. Thus, the sample was ascertained on the basis of kinship and not on disease status. The 20 founding couples were related to each other through previous generations; hence study participants are members of a single large extended pedigree. Analysis of this highly complex pedigree struc- ture by PEDSTATS [13] revealed 1,444 founders and 5,148 non-founders, with 1,617 consanguineous marriages and 3,060 sibships (average size of the sibships is 2). The number of each type of relative pair is reported in Table 1. The mean of the inbreeding coefficient is $0.003 \pm 0.00008$ (range 0-0.066), which indicates that most of the consanguineous marriages are between relations of at least the fifth degree.

Members of the pedigree were invited to our research centre, which is located in the vicinity. Participants were sent questionnaires and consent forms prior to arrival at the centre and interviewed upon arrival, thus ensuring informed consent. During a one-day visit, each participant underwent several examination blocks that included a medical history obtained by a physician, venipuncture for biochemistry, and blood pressure and anthropometric measurements, including those by DEXA.

\section{Assessment of study variables}

Participants were asked to bring all current medications for registration. This information was used to create variables for antidiabetic (insulin and oral hypoglycaemic) treatment, as well as antihypertensive, lipid-lowering and potentially glucose-increasing agents (i.e. oral glucocorticoids, thyroid hormone, $\beta$-agonists, thiazides and $\beta$-blockers).

Participants were asked to fast for at least $8 \mathrm{~h}$ prior to blood withdrawal. Blood samples were then sent to the local laboratory for standard plasma glucose and serum lipid measurements, which were made using a Synchron LX 20 Systems analyser (Beckman Coulter, Fullerton, CA, USA).

Blood pressure in the right arm was measured while sitting, using an automated device (OMRON HEM-711; OMRON Healthcare, Vernon Hills, IL, USA) after at least $5 \mathrm{~min}$ of rest. The average of two measurements taken 5 min apart was used for analysis. Pulse pressure and mean

Table 1 Number of relative pairs within pedigree from the ERF study

\begin{tabular}{lcc}
\hline Characteristic & Whole pedigree $(n)$ & Phenotyped subset $(n)$ \\
\hline Pedigree members & 6,592 & $852^{\mathrm{a}}$ \\
Founders & 1,444 & 140 \\
Non-founders & 5,148 & 712 \\
Relative pairs & & \\
Parent-offspring & 10,296 & 326 \\
Sibling-sibling & 3,876 & 468 \\
Grandparent & 16,298 & 26 \\
Avuncular & 14,902 & 785 \\
Half-siblings & 449 & 22 \\
Cousins & 17,364 & 1,998 \\
\hline
\end{tabular}

Number of relative pairs given in the third column denotes the number of pairs for which quantitative phenotypes are available for both members of the pair

${ }^{a}$ Although only 852 were phenotyped, the structure of the whole pedigree including 6,592 individuals was used as input for heritability analyses 
arterial pressure were calculated as possible surrogate measures.

Anthropometric and DEXA measurements were made with participants in their underwear and without shoes. Using soft tape, with the subject in the standing position, waist circumference was measured midway between the lower costal margin and the iliac crest, while hip circumference was taken at the hip's widest diameter. Waist-to-hip ratio (WHR) was then computed. Height and weight were also measured. BMI was calculated as weight in kilograms divided by the square of height in metres. All DEXA scans were performed in a calibrated Prodigy total body fanbeam densitometer and analysed with enCORE 2002 software version 6.70.021 (GE Lunar, Madison, WI, USA). Other parameters estimated by DEXA included fat and lean mass for total body, trunk and legs. The following indices were then determined: \%fat mass $(\mathrm{FM})=\mathrm{FM} /$ (FM + lean mass), both for total body and for body regions; lean mass index $(\mathrm{LMI})=$ total lean mass (in $\mathrm{kg}) /$ (height in $\mathrm{m})^{2}$; fat mass index (FMI) = total FM (in $\mathrm{kg}$ )/ (height in $\mathrm{m}^{2}$; total/trunk fat ratio; total/legs fat ratio; and trunk/legs fat ratio $(\mathrm{Tr} / \mathrm{l})$.

Of 951 phenotyped participants (as of September 2003), 905 had FPG measurements, 47 of whom were identified as using antidiabetic medications. Inclusion of participants who were on antidiabetic treatment resulted in a severely skewed distribution for both FPG and triglyceride (Trig) variables. Therefore, analysis was restricted to 852 participants without antidiabetic treatment, who also had complete measurements on body composition, lipids and blood pressure.

\section{Statistical analysis}

SPSS 11.0.1 (SPSS, Chicago, IL, USA) was used for descriptive and regression analyses. The large number of measurements and participants permitted adjustment for multiple covariates in the analyses. Apart from lipid, body and blood pressure measurements, information on smoking and medications that may potentially increase glucose levels were available. Because of its non-linear relationship with glucose, age was categorised into five groups with 15year intervals. Because the distribution of FPG was not normal, log normal (ln)-transformed FPG was regressed on each covariate. Multiple regression analysis was then performed with all the covariates in the model. Covariates were also grouped into blocks, i.e. body composition, blood pressure and lipids. For each block the most significant independent predictors were chosen on the basis of existing literature and on multivariable regression analyses done for each block separately and with all blocks together. By hierarchical modelling and multiple linear regression with different covariate subsets, the following covariates were identified as significant: $\operatorname{lnFPG} \sim \mu+\beta_{\text {age }}$ age $+\beta_{\mathrm{LMI}} \mathrm{LMI}+\beta_{\mathrm{FMI}} \mathrm{FMI}+\beta_{\operatorname{Tr} / 1} \operatorname{Tr} / 1+\beta_{\mathrm{SBP}}$ systolic blood pressure (SBP) $+\beta_{\text {Trig }}$ Trig $+\beta_{\mathrm{TC} / \mathrm{HDL}}$ total cholesterol/ HDL-cholesterol ratio (TC/HDL). Sex was kept as a covariate in the final model. Potentially glucose-increasing medication and smoking did not affect FPG significantly and were therefore excluded from further analyses.

In heritability analysis, we assumed a standard model describing the expectation of the quantitative trait, $Q_{i j}$, of the $i$ th person who is an offspring of the $j$ th mother as

$Q_{i j}=\mu+\sum_{k} \beta_{k} C_{k i}+a_{i}+s_{j}+\varepsilon_{i j}$,

where $\mu$ is the population mean, $C_{k i}$ is the value of $k$ th covariate (fixed effect) for person $i, \beta_{k}$ is the regression coefficient, $a_{i}$ is the additive genetic effect, $s_{j}$ is the sibship effect (which is discussed below) and $\varepsilon_{i j}$ is the residual (environmental) effect. It is assumed that random additive genetic, sibship and environmental effects are normally distributed with mean zero and variances equal to $\sigma_{a}^{2}, \sigma_{s}^{2}$ and $\sigma_{e}^{2}$, respectively. The variance-covariance structure is given by

$\operatorname{Cov}(i, j)=\left\{\begin{array}{cc}\sigma_{a}^{2}+\sigma_{s}^{2}+\sigma_{e}^{2}, & \text { if } i=j \\ 2 \phi_{i j} \sigma_{a}^{2}+\eta_{i j} \sigma_{s}^{2}, & \text { if } i \neq j\end{array}\right.$,

where $\phi_{i j}$ is the coefficient of kinship between individuals $i$ and $j$, and $\eta_{i j}$ is equal to one if $i$ and $j$ share the same mother (and are therefore sibs or maternal half-sibs). In the above formulation, the narrow-sense heritability is defined as $h^{2}=$ $\sigma_{a}^{2} /\left(\sigma_{a}^{2}+\sigma_{e}^{2}\right)$ when the sibship effect is not considered, and by $h^{2}=\sigma_{a}^{2} /\left(\sigma_{a}^{2}+\sigma_{s}^{2}+\sigma_{e}^{2}\right)$ when the sibship effect is included in the analysis. The proportion of variance due to the sibship effect was computed with the formula $S=\sigma_{s}^{2} /$ $\left(\sigma_{a}^{2}+\sigma_{s}^{2}+\sigma_{e}^{2}\right)$.

The sibship effect estimates phenotypic similarities induced in the progeny of the same mother. This effect is a combination of effects induced by shared early-life environment and dominance genetic effects. It may be argued that this effect is better termed 'maternal'. However, one widely accepted definition of the maternal effect is a mitochondrial effect, which we did not directly estimate in our model. Moreover, because of the small number of halfsibs in our sample (Table 1) and the non-delineation of household effects in our data set, the effect due to sharing the same mother is almost indistinguishable from the sibship effect. Therefore, for clarity we refer to it as the 'sibship effect'.

To estimate the linear mixed model described above, the ASReml 1.10 program (NSW Agriculture Biometrics, NSW, Australia; URL http://www.vsn-intl.com/ASReml/) for restricted maximum likelihood analysis was used. ASReml calculates the inverse relationship matrix from the pedigree structure. In this study the entire pedigree including 6,592 members was analysed as a single unit; each analysis took only a few minutes to run since the software was developed for analysis of agricultural data and is capable of handling extremely large experimental data sets. Because the ASReml manual recommends that all covariates have a normal distribution, values of LMI, 
FMI, Tr/1, SBP, Trig and TC/HDL were ln-transformed to improve normality. ASReml was used to fit a linear mixed model to the data, with the covariate traits as fixed effects and the genetic and sibship effects as random effects.

The statistical significance of random effects (heritability and sibship effect) was assessed using the likelihood ratio test. Under the null hypothesis, twice the difference between the residual log likelihood values from the nesting models with the same fixed effects follows a $\chi^{2}$ distribution, with the number of degrees of freedom equal to the difference in the number of estimated parameters. Due to the nature of restricted maximum likelihood, the likelihood ratio test cannot be used to assess the significance of fixed effects. However, the square of the ratio between the estimate and the standard error, which follows a $\chi^{2}$ distribution, can be used for testing. All heritabilities are reported as the estimate $h^{2} \pm \mathrm{SE}$.

\section{Results}

At the time of analysis, measurements of FPG, body composition, blood pressure and lipids were complete for 852 individuals (Table 1). The mean age of participants was $51.9 \pm 14.0$ years SD (median 52.4, range 18-85). Table 2 shows the characteristics of the study population. Blood pressure and lipid measurements, except for HDL cholesterol, were significantly different among age groups (data not shown). Males constituted $40 \%$ of the sample. Sex-related differences were found for FPG, age, BMI, WHR, total fat mass, LMI, FMI, fat mass ratios, HDL cholesterol, Trig, TC/ HDL and blood pressure (not all data shown). To account for these sex differences, sex was maintained as a covariate in the models for heritability estimation, even though sex was not significant in multiple regression analyses with all covariates included and with the covariates grouped into blocks.

The heritability estimate for FPG was $36.6 \pm 8.6 \%$ in the sample of participants without antidiabetic medications, when adjusted for age and sex (Table 3). To study the relationship between metabolic risk factors and FPG, adjustment for these factors was made and the corresponding changes in heritability estimates were noted. Adjustment for body composition parameters (LMI, FMI and Tr/l) increased the heritability of FPG $(43.2 \pm 8.9 \%)$. In contrast, adding SBP or Trig and TC/HDL to the model produced a slight reduction in heritability. Using the full model, which includes variables that represent each component of the metabolic syndrome, we obtained a heritability estimate of $42.8 \pm 9.0 \%$.

Excluding patients with antidiabetic treatment eliminated more extreme glucose levels. When subjects with antidiabetic medication were included in the analysis $(n=905)$, the heritability estimate did not change substantially after correction for treatment, using all models. For this analysis, the categorical variable for antidiabetic treatment was a significant covariate.

It has been suggested previously that early-life environment may contribute to the homeostatic control of blood glucose levels in adulthood [14]. Therefore, the sibship effect was included in the model as a random effect. Inclusion of the sibship effect reduced heritability estimates for all models, the age- and sex-adjusted and fully adjusted estimates being $28.3 \pm 10.6$ and $24.9 \pm 11.6 \%$, respectively (Table 3). Despite the fact that heritability estimates were lower, they remained significant at $p<0.05$ for all models.

In models with the sibship effect, the regression coefficients for covariates were virtually the same as in the initial analysis not including sibship effect (data not shown). Age was a significant covariate in all the models tested, with or without the sibship effect $(p<0.05)$. Likelihood ratio testing showed that addition of the sibship effect to the model was statistically significant only when body composition covariates were added into the model: sibship effect $p$ values were significant only for model 2 $(p<0.01$, age, sex and body composition as covariates) and model 5 ( $p<0.01$, full model) in Table 3. Interestingly, for

Table 2 Clinical characteristics of 852 participants in the ERF study

\begin{tabular}{|c|c|c|c|c|c|c|c|}
\hline \multirow[t]{2}{*}{ Trait $^{\mathrm{a}}$} & \multicolumn{4}{|l|}{ Overall } & \multicolumn{3}{|l|}{ By sex } \\
\hline & Mean \pm SE & $\ln$ Mean $\pm \mathrm{SE}^{\mathrm{b}}$ & Skewness ${ }^{c}$ & Kurtosis $^{c}$ & Men & Women & $p$ value \\
\hline$n$ & 852 & - & - & - & 345 & 507 & - \\
\hline FPG (mmol/l) & $4.6 \pm 0.03$ & $1.5 \pm 0.006$ & 1.3 & 4.1 & $4.8 \pm 0.04$ & $4.5 \pm 0.03$ & $<0.001$ \\
\hline LMI $\left(\mathrm{kg} / \mathrm{m}^{2}\right)$ & $16.9 \pm 0.09$ & $2.8 \pm 0.005$ & 0.4 & -0.4 & $19.1 \pm 0.10$ & $15.4 \pm 0.06$ & $<0.001$ \\
\hline $\mathrm{SBP}(\mathrm{mmHg})$ & $141.0 \pm 0.75$ & $4.9 \pm 0.005$ & 0.9 & 1.0 & $145.2 \pm 1.10$ & $138.1 \pm 0.77$ & $<0.001$ \\
\hline Trig $(\mathrm{mmol} / \mathrm{l})$ & $1.4 \pm 0.03$ & $0.2 \pm 0.02$ & 2.0 & 7.4 & $1.6 \pm 0.05$ & $1.2 \pm 0.02$ & $<0.001$ \\
\hline TC/HDL & $4.7 \pm 0.05$ & $1.5 \pm 0.01$ & 0.5 & 0.1 & $5.1 \pm 0.08$ & $4.3 \pm 0.04$ & $<0.001$ \\
\hline
\end{tabular}

The following variables were the most significant predictors after multiple regression analyses: age, LMI, FMI, Tr/l, SBP, Trig and TC/HDL ${ }^{\text {a } F P G ~ f a s t i n g ~ p l a s m a ~ g l u c o s e ; ~} L M I$ lean mass index; FMI fat mass index; Tr/l trunk/legs fat ratio; SBP systolic blood pressure; Trig triglycerides; TC/HDL ratio total cholesterol/HDL

$\mathrm{b}$ ln Mean average of ln-transformed values

${ }^{\mathrm{c}}$ Skewness and kurtosis reported are for the distribution of original non-transformed values

${ }^{\mathrm{d}} p$ value by $t$ test using $\ln$-transformed values 
Table 3 Heritability of ln-transformed fasting glucose $\left(h^{2}{ }_{\operatorname{lnFPG}} \pm \mathrm{SE}\right)$

\begin{tabular}{|c|c|c|c|c|c|}
\hline \multirow[t]{2}{*}{ Parameter $^{\mathrm{a}}$} & \multicolumn{5}{|l|}{ Model $^{\mathrm{b}}$} \\
\hline & 1 & 2 & 3 & 4 & 5 \\
\hline \multicolumn{6}{|l|}{ Without sibship effect } \\
\hline$\mu$ & $1.42 \pm 0.02$ & $0.62 \pm 0.15$ & $0.54 \pm 0.19$ & $1.42 \pm 0.02$ & $0.07 \pm 0.23$ \\
\hline$\beta_{\text {age30-45 }}$ & $0.06 \pm 0.02$ & $0.04 \pm 0.02$ & $0.06 \pm 0.02$ & $0.06 \pm 0.02$ & $0.04 \pm 0.02$ \\
\hline$\beta_{\text {age45-60 }}$ & $0.14 \pm 0.02$ & $0.09 \pm 0.02$ & $0.12 \pm 0.02$ & $0.12 \pm 0.02$ & $0.08 \pm 0.02$ \\
\hline$\beta_{\text {age60-75 }}$ & $0.19 \pm 0.02$ & $0.14 \pm 0.02$ & $0.16 \pm 0.02$ & $0.18 \pm 0.02$ & $0.12 \pm 0.02$ \\
\hline$\beta_{\text {age75+ }}$ & $0.13 \pm 0.03$ & $0.10 \pm 0.03$ & $0.09 \pm 0.03$ & $0.12 \pm 0.03$ & $0.07 \pm 0.03$ \\
\hline$\beta_{\mathrm{sex}}$ & $-0.05 \pm 0.01$ & $-0.009 \pm 0.02$ & $-0.05 \pm 0.01$ & $-0.04 \pm 0.01$ & $-0.008 \pm 0.02$ \\
\hline$\beta_{\mathrm{lnLMI}}$ & {$[0]$} & $0.23 \pm 0.05$ & {$[0]$} & {$[0]$} & $0.24 \pm 0.05$ \\
\hline$\beta_{\text {lnFMI }}$ & {$[0]$} & $0.07 \pm 0.01$ & {$[0]$} & {$[0]$} & $0.06 \pm 0.01$ \\
\hline$\beta_{\operatorname{lnTr} / 1}$ & {$[0]$} & $0.03 \pm 0.02$ & {$[0]$} & {$[0]$} & $0.02 \pm 0.02$ \\
\hline$\beta_{\operatorname{lnSBP}}$ & {$[0]$} & {$[0]$} & $0.18 \pm 0.04$ & {$[0]$} & $0.11 \pm 0.04$ \\
\hline$\beta_{\operatorname{lnTrig}}$ & {$[0]$} & {$[0]$} & {$[0]$} & $0.04 \pm 0.01$ & $0.02 \pm 0.01$ \\
\hline$\beta_{\mathrm{InTC} / \mathrm{HDL}}$ & {$[0]$} & {$[0]$} & {$[0]$} & $-0.009 \pm 0.02$ & $0.03 \pm 0.02$ \\
\hline$h_{\operatorname{lnFPG}}^{2}(\%)$ & $36.6 \pm 8.6$ & $43.2 \pm 8.9$ & $35.6 \pm 8.7$ & $37.7 \pm 8.8$ & $42.8 \pm 9.0$ \\
\hline$\sigma_{\rho}^{2}$ & 0.014 & 0.012 & 0.014 & 0.013 & 0.011 \\
\hline$p$ value, $h_{\text {InFPG }}^{2}$ & $4 \times 10^{-6}$ & $1 \times 10^{-6}$ & $1 \times 10^{-5}$ & $6 \times 10^{-6}$ & $1 \times 10^{-6}$ \\
\hline \multicolumn{6}{|l|}{ With sibship effect } \\
\hline$h^{2}{ }_{\mathrm{InFPG}}(\%)$ & $28.3 \pm 10.6$ & $26.4 \pm 11.4$ & $26.1 \pm 10.7$ & $28.5 \pm 10.8$ & $24.9 \pm 11.6$ \\
\hline$\% \mathrm{~S}$ (sibship effect) & $12.3 \pm 7.0$ & $22.3 \pm 7.3$ & $13.4 \pm 7.1$ & $13.7 \pm 7.2$ & $22.8 \pm 7.4$ \\
\hline$\sigma_{e}^{2}$ & 0.013 & 0.010 & 0.013 & 0.013 & 0.011 \\
\hline$p$ value, $\mathrm{S}^{\mathrm{c}}$ & 0.08 & 0.001 & 0.06 & 0.06 & 0.001 \\
\hline
\end{tabular}

${ }^{a} \mu$ Intercept; $L M I$ lean mass index; FMI fat mass index; $T r / l$ trunk/legs fat ratio; SBP systolic blood pressure; Trig triglyceride; $T C / H D L$ ratio total cholesterol/HDL; $\sigma_{e}^{2}$ environmental variance. Age group 15-30 is reference category

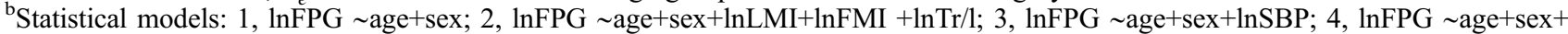
$\ln$ Trig $+\ln$ TC/HDL; $5, \ln F P G \sim$ age + sex $+\ln L M I+\operatorname{lnFMI}+\ln T r / 1+\operatorname{lnSBP}+\ln$ Trig $+\operatorname{lnTC} / \mathrm{HDL}$ (full model). [0] Coefficient set to zero/variable not included in model

${ }^{c} p$ values were derived using the likelihood ratio test

the same models with body composition parameters, the effect of sex was non-significant. LMI, FMI, SBP and Trig, but not $\mathrm{Tr} / \mathrm{l}$ and $\mathrm{TC} / \mathrm{HDL}$, reached statistical significance when included in the analysis.

\section{Discussion}

Despite many studies on the genetics of the metabolic syndrome and its components, the relative roles of environment and genetics in this syndrome remain unclear. In our study, adjustment for metabolic risk factors increased rather than decreased heritability estimates of FPG. This may mean that a significant portion of the variance in glucose levels is due to genes that act through pathways that are independent of those controlling body composition, blood pressure and lipid levels.

After adjustment for age, sex, metabolic risk factors and the sibship effect, the heritability of FPG in this sample was $25 \%$. The sibship effect was significant in models that included body composition measurements as covariates (model 2 and the full model model 5). In these models, the sibship effect explained an amount of variability in glucose levels that was similar to that explained by the additive genetic component. Modelling of the sibship effect in the absence of genetic marker data does not allow distinction between the contributions of shared early-life environment and dominance genetic effects. These environmental effects, which may contribute to the shared environment of siblings, are likely to be mediated by maternal influences (e.g. intrauterine environment and neonatal feeding) and lifestyle effects (e.g. diet and physical activity).

The intrauterine environment has been shown to affect adult glucose metabolism. In human studies of middleaged subjects whose mothers were exposed to famine during pregnancy, significant glucose intolerance was noted [14]. In rat studies, adults with fetal protein malnutrition had decreased insulin release but normal peripheral uptake in muscle [15]. This supports the hypothesis that impaired development of beta cell function due to early-life food deprivation predisposes to impaired glucose tolerance in adulthood, particularly after later adaptation of sedentary and/or hyperphagic lifestyles [16].

Glucose levels increased significantly with age, as shown by the age coefficients in our analysis (Table 3 ). However, the progressive increase in glucose levels with age stopped at the age range $50-75$; those aged $\geq 75$ had lower glucose levels (Table 3). This may be due to a healthy survivor effect, such that those who survived beyond age 75 may constitute a special population that is characterised by a specific blend of genetic and environmental factors which contribute to longevity. Though sex 
affects variability in glucose levels, sex differences became non-significant after adjustment for body composition.

Unexpectedly, among the body composition parameters, LMI had the highest impact, both in terms of significance and the effect size on FPG. Several studies have demonstrated that visceral or abdominal obesity and high fat mass, more than BMI, are associated with insulin resistance [17]. The strong positive association between LMI and FPG deserves further investigation.

Genetically isolated populations are characterised by inbreeding and increased genetic drift and thus may deviate in their genetic composition from the general population. Moreover, in such populations there is an increased chance of loss or fixation of some genetic variants, thus potentially leading to lower genetic diversity and consequently lower heritability. However, in a recent study [18] we have shown that effects of drift are relatively small in our genetically isolated population and the chance of losing an allele is very small, unless it had a very low frequency in the original population. Thus, our population should not deviate much from the general population in its genetic composition and we do not expect that our heritability estimates would differ substantially from those in the general Dutch population.

Recent studies on fasting glucose among populations of European descent have found linkage to different chromosomes. In the Finnish population, where the heritability of fasting glucose was estimated to be $8.4 \%$ after correction for ascertainment, elevated fasting glucose was suggestively linked to chromosome 3 [19]. In another population of European Caucasian descent, the heritability of fasting glucose was $34 \%$, but only suggestive linkage to chromosome 10q and weak linkage to 1q were found [20]. Given a heritability estimate of at least $25 \%$ for FPG, further linkage studies in the ERF population could well find genes that affect FPG levels and possibly play a role in outbred populations.

In conclusion, the heritability of FPG in this young, genetically isolated population is at least $25 \%$. Sibship variance, which includes dominance genetic effects and common effects of the early-life environment, contributed a similar amount of variability in glucose levels after adjustment for body composition. A significant proportion of the variance in FPG level is controlled by genes that act independently of genes that influence body composition, blood pressure and lipids.

Acknowledgements The authors would like to thank the participants in the study. This study was made possible through grants from the Netherlands Organization for Scientific Research (NWO), Centre for Medical Systems Biology (CMSB), the Netherlands Kidney Foundation and the Netherlands Heart Foundation.

\section{References}

1. King H, Aubert RE, Herman WH (1998) Global burden of diabetes, 1995-2025. Diabetes Care 21:1414-1431
2. Wilson PWF, Grundy SM (2003) The metabolic syndrome. Practical guide to origins and treatment: part I. Circulation 108:1422-1425

3. Goldin LR, Camp NJ, Keen KJ et al (2003) Analysis of metabolic syndrome phenotypes in Framingham Heart Study families from Genetic Analysis Workshop 13. Genet Epidemiol 25(Suppl 1):S78-S89

4. Barker DJ (1997) Maternal nutrition, fetal nutrition, and disease in later life. Nutrition 13:807-813

5. Zhu S, St-Onge MP, Heshka S, Heymsfield SB (2004) Lifestyle behaviors associated with lower risk of having the metabolic syndrome. Metabolism 53:1503-1511

6. Falconer DS (1981) Introduction to quantitative genetics, 2nd edn. Longman, New York

7. Poulsen P, Kyvik KO, Vaag A, Beck-Nielsen H (1999) Heritability of type II (non-insulin-dependent) diabetes mellitus and abnormal glucose tolerance-a population-based twin study. Diabetologia 42:139-145

8. Snieder H, Boomsma DI, van Doornen LJP, Neale MC (1999) Bivariate genetic analysis of fasting insulin and glucose levels. Genet Epidemiol 16:426-446

9. Snieder H, Sawtell PA, Ross L, Walker J, Spector TD, Leslie $\mathrm{RD}$ (2001) HbA(1c) levels are genetically determined even in type 1 diabetes: evidence from healthy and diabetic twins. Diabetes 50:2858-2863

10. Watanabe RM, Valle T, Hauser ER et al (1999) Familiality of quantitative metabolic traits in Finnish families with noninsulin-dependent diabetes mellitus. Finland-United States Investigation of NIDDM Genetics (FUSION) Study investigators. Hum Hered 49:159-168

11. Mills GW, Avery PJ, McCarthy MI et al (2004) Heritability estimates for beta cell function and features of the insulin resistance syndrome in UK families with an increased susceptibility to type 2 diabetes. Diabetologia 47:732-738

12. Freeman MS, Mansfield MW, Barrett JH, Grant PJ (2002) Heritability of features of the insulin resistance syndrome in a community-based study of healthy families. Diabet Med 19:994-999

13. Wigginton JE, Abecasis GR (2005) PEDSTATS: descriptive statistics, graphics and quality assessment for gene mapping data. Bioinformatics 21:3445-3447

14. Ravelli ACJ, van der Meulen JHP, Michels RPJ et al (1998) Glucose tolerance in adults after prenatal exposure to famine. Lancet 351:173-177

15. Holemans K, Aerts L, Van Assche FA (2003) Lifetime consequences of abnormal fetal pancreatic development. J Physiol 547:11-20

16. Hales CN, Barker DJP, Clark PMS et al (1991) Fetal and infant growth and impaired glucose tolerance at age 64. BMJ 303:1019-1022

17. Karelis AD, St-Pierre DH, Conus F, Rabasa-Lhoret R, Poehlman ET (2004) Metabolic and body composition factors in subgroups of obesity: what do we know? J Clin Endocrinol Metab 89:2569-2575

18. Pardo LM, MacKay I, Oostra B, van Duijn CM, Aulchenko YS (2005) The effect of genetic drift in a young genetically isolated population. Ann Hum Genet 69:288-295

19. Watanabe RM, Ghosh S, Langerfeld CD et al (2000) The Finland-United States Investigation of Non-Insulin-Dependent Diabetes Mellitus Genetics (FUSION) Study. II. An autosomal genome scan for diabetes-related quantitative-trait loci. Am J Hum Genet 67:1186-1200

20. Meigs JB, Panhuysen CIM, Myers RH, Wilson PWF, Cupples LA (2002) A genome-wide scan for loci linked to plasma levels of glucose and $\mathrm{HbA}_{1 \mathrm{c}}$ in a community-based sample of Caucasian pedigrees. The Framingham offspring study. Diabetes 51:833-840 